\title{
Barragens de mineração na Amazônia: potencial poluidor- degradador ambiental e ameaça à saúde coletiva
}

O objetivo deste artigo foi realizar um estudo sobre barragens de mineração na Amazônia, focalizando aquelas localizadas em Parauapebas, que é o principa município minerador do Brasil. Foram consideradas as barragens classificadas com Dano Potencial Associado Alto - DPAA, para analisar os possíveis impactos ambientais, danos à saúde das populações circundantes, bem como a existência efetiva do plano de ação de emergência em caso de sinistro. Justifica-se este tema motivado pelos últimos desastres ocorridos por rompimento de barragens no Brasil, ressaltando a necessidade de melhorias das condições de segurança dessas estruturas e também de novos métodos e práticas na mineração que conciliem a eficiência econômica com a eficiência ambiental. A pesquisa desenvolveu-se sob vasto arcabouço teórico, bem como de dados adquiridos em órgãos oficiais brasileiros. Os resultados apontam que apesar das tragédias recentes ocorridas no país, a fiscalização para os cumprimentos legais e atendimento às normas estabelecidas ainda é limitada e muito dependente do monitoramento das próprias mineradoras, o que eleva o potencial de degradação ao ambiente e a saúde coletiva face à exploração de minério. Mediante análise da legislação brasileira fo percebido que está possui lacunas para o consentimento de licenciamentos frágeis e com precário poder fiscalizatório do Estado. Há ainda a existência tacanha e ineficaz do Plano de Ação de Emergência para Barragem de Mineração-PAEBM, cuja obrigatoriedade por lei é recente e que possui dificuldades para ser executado com eficiência, haja vista que atualmente, no estado do Pará, inexistem condições objetivas e eficazes de garantir a segurança destas barragens, visto que há um intrincado cenário político e econômico desfavorável ao cumprimento das normas, aliado ao exíguo número de fiscais capacitados para atuar no controle e fiscalização, de forma preventiva, para garantir a segurança dessas estruturas.

Palavras-chave: Rejeitos minerais; Degradação ambiental; Responsabilidade socioambiental; Saúde pública.

\section{Mining dams in the Amazon: potential environmental pollutor- degrader and threat to collective health}

\begin{abstract}
The purpose of this article was to conduct a study on mining dams in the Amazon, focusing on those located in Parauapebas, which is the main mining municipality in Brazil. Dams classified as High Potential Damage Associated - HPDA were considered to analyze the possible environmental impacts, damage to the health of surrounding populations, as well as the effective existence of the emergency action plan in the event of an accident. This issue is motivated by last disasters that occurred due to collapse of dams in Brazil, highlighting the need to improve the safety conditions of these structures and also new methods and practices in mining that combine economic efficiency with environmental efficiency. The research was developed under a vast theoretical framework, as well as data acquired in official Brazilian Government agencies. The results show that despite the recent tragedies that have occurred in the country, the inspection for legal compliance with established standards is still limited and very dependent on monitoring of mining companies themselves, which increases the potential for degradation to the environment and collective health due to ore exploration. Upon analysis of the Brazilian legislation, it was noticed gaps that result fragile licensing processes and with poor inspection capacity of the State. There is also the inadequate and ineffective existence of the Emergency Action Plan for Mining Dam-EAPMD, whose mandatory by law is recent and which has difficulties to be carried out efficiently, given that currently, in the state of Pará, there are no effective conditions to guarantee the safety of these dams, since there is an intricate political and economic scenario unfavorable to compliance with the rules, coupled with the insufficient number of inspectors trained to act in control and inspection, in a preventive manner, to guarantee the safety of these structures.
\end{abstract}

Keywords: Mineral tailings; Ambient degradation; Social and environmental responsibility; Public health.

Topic: Desenvolvimento, Sustentabilidade e Meio Ambiente

Reviewed anonymously in the process of blind peer.

Mônica Moraes Ribeiro (it)

Universidade do Estado do Pará, Brasil

http://lattes.cnpq.br/0755419846048931

http://orcid.org/0000-0001-6310-557X

profa.monica.mr@gmail.com

Thamires Beatriz dos Santos Caitano (id

Universidade do Estado do Pará, Brasil

http://lattes.cnpq.br/4649556839610299

http://orcid.org/0000-0002-7538-4366

thamirescaitano@hotmail.com

Isabelle Moraes Ribeiro (iD

Universidade de Brasília, Brasil

http://lattes.cnpq.br/2212935604490810

http://orcid.org/0000-0002-8211-265X

isabellemoraes.unb@gmail.com

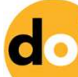

DOI: $10.6008 / C B P C 2179-6858.2020 .006 .0043$
Received: 05/10/2020

Approved: 26/11/2020

\author{
Altem Nascimento Pontes (iD \\ Universidade do Estado do Pará, Brasil \\ http://lattes.cnpq.br/5993352890364998 \\ http://orcid.org/0000-0002-9001-4603 \\ altempontes@hotmail.com
}

\section{Referencing this:}

RIBEIRO, M. M.; CAITANO, T. B. S.; RIBEIRO, I. M.; PONTES, A. N.. Barragens de mineração na Amazônia: potencial poluidor-degradador ambiental e ameaça à saúde coletiva. Revista Ibero Americana de Ciências Ambientais, v.11, n.6, p.532-544, 2020. DOI: http://doi.org/10.6008/CBPC2179-6858.2020.006.0043 


\section{INTRODUÇÃO}

Tragédias ambientais recentes no Brasil envolvendo barragens de contenção de rejeitos de mineração e de resíduos industriais vêm suscitando questionamentos quanto à permissão para a utilização dessas estruturas, que crescem com o aumento da produção mineral industrial no país (GARCIA et al., 2017; MORAES et al., 2019).

Em 5 de novembro de 2015 ocorreu o acidente em Mariana, município brasileiro localizado no estado de Minas Gerais, na região sudeste do Brasil, onde mais de 70 milhões de metros cúbicos de lama de rejeitos da mineração de ferro vazaram após o rompimento da barragem de Fundão pertencente à Samarco, uma empresa joint-venture da multinacional Vale S.A e da anglo-australiana BHP Billiton (MEYER et al., 2020). Nesta tragédia, com vários mortos, desaparecidos e centenas de pessoas desabrigadas, revelou-se o desmesurado passivo ambiental e a vulnerabilidade das comunidades que vivem em seu entorno e que ainda esperam por reparação (FREITAS et al., 2016).

Pouco mais de três anos após o desastre de Mariana, em 25 de janeiro de 2019, aconteceu outra tragédia por rompimento de barragens, está ocorrida na mina do Córrego do Feijão em Brumadinho (MG), da empresa Vale S.A, o que resultou em um dos maiores desastres com rejeitos de mineração no Brasil com a morte de centenas de pessoas e onde dezenas de pessoas continuam desaparecidas (HINMAN, 2019). Os dois lamentáveis episódios nas duas cidades de Minas Gerais foram causados pelo mesmo motivo que foi o rompimento das barragens de contenção de resíduos e detritos, fruto da elaboração e refino do minério de ferro (DIOS et al., 2019).

Esses crimes ambientais causaram poluição no ecossistema por rejeitos minerais, com contaminação hídrica, destruição de área florestal considerada como área de preservação permanente e sérios danos à saúde coletiva (AIRES et al., 2018; BURRITT et al., 2018). Tais fatos marcaram, de forma indelével, a história do Brasil, visto que causaram mortes de centenas de pessoas, efeitos negativos sobre a saúde coletiva e grande impacto degradador no ambiente cujos danos são imensuráveis e de difícil gestão (CARMO et al., 2017; SEVERO et al., 2019).

Após a configuração desse grave panorama ambiental nacional da atualidade, os interesses econômicos da indústria mineral avultaram, a pleno vapor, para a região amazônica (HAURADOU et al., 2019; NEGRÃO, 2019). A Amazônia ocupa posição de destaque na produção e exportação de bens minerais. Dados do Instituto Brasileiro de Mineração - IBRAM e da Agência Nacional de Mineração - ANM indicam a atividade da mineração como propulsora de crescimento econômico na região norte, destacando o município de Parauapebas no cenário minerário nacional e internacional (ANM, 2019).

Diante desses fatos, para este estudo, levantou-se alguns questionamentos sobre os fatores atuais que potencializam riscos de acidentes em barragens classificadas pela ANM com Dano Potencial Associado Alto-DPAA e que estão localizadas em Parauapebas, sobre os impactos no ambiente e saúde das populações circundantes, bem como sobre a existência de um plano de emergência efetivo em caso de desastre.

Apresentou-se então a hipótese de que a fragilidade institucional no cumprimento da legislação 
(PELICICE, 2019); associada à pressão econômica para a exploração e exportação de commodities (GEVORKYAN, 2019) pode elevar a possibilidade de acidentes na indústria da mineração com barragens nesta região (FREITAS et al., 2019). A hipótese justifica-se pelos episódios de desastres recentes ocorridos no Brasil, bem como pela condição geopolítica da Amazônia que, historicamente, atua como província extrativista mineral, sob os ditames do mercado internacional com seu ímpeto de crescimento econômico perpétuo e a qualquer custo (SOMMER et al., 2019).

Em face ao exposto, o objetivo deste artigo foi realizar um estudo sobre as barragens de mineração na Amazônia, focalizando aquelas localizadas em Parauapebas, que é o principal município minerador do Brasil, considerando as barragens que foram classificadas, segundo dados da ANM, com DPAA, para analisar os possíveis impactos ambientais, os danos à saúde das populações circundantes, bem como sobre a existência efetiva do plano de ação de emergência em caso de desastre.

\section{DISCUSSÃO TEÓRICA}

\section{As barragens da mineração}

Barragens podem ser conceituadas como qualquer estrutura em um curso permanente ou temporário de água para fins de contenção ou acumulação de substâncias líquidas ou de misturas de líquidos e sólidos, compreendendo o barramento e as estruturas associadas (THOMÉ et al., 2017). As barragens de rejeitos na atividade mineral industrial são usadas para depositar os resíduos e a água gerados a partir do beneficiamento do minério (SOUZA, 2019).

O beneficiamento do minério consiste em separar o material valioso presente nos minerais do restante que não tem valor comercial, sendo rejeitado pelo processo produtivo e represado no meio ambiente (SANT'ANA FILHO et al., 2017). As barragens existem para que haja a transformação do recurso mineral em um produto, com valor econômico que atenda às necessidades do mercado internacional (SCHORN et al., 2019).

Desta forma, é de suma importância compreender e dispor de dados e informações técnicas sobre essas construções (ALMEIDA et al., 2020). É fundamental também conhecer a legislação existente no País, para analisar as lacunas e possibilidades sobre as barragens de rejeitos minerários, sobre os direitos das populações que vivem no entorno desses empreendimentos, bem como apreender a dimensão social dos impactos causados pela mineração nessas comunidades (GEROTTO et al., 2019; SOUZA et al., 2019).

\section{Legislação vigente no Brasil}

A Portaria № 70.389, de 17 de maio de 2017, criou o Cadastro Nacional de Barragens de Mineração, o Sistema Integrado de Gestão em Segurança de Barragens de Mineração onde estabelece a periodicidade de execução ou atualização, a qualificação dos responsáveis técnicos, o conteúdo mínimo e o nível de detalhamento do Plano de Segurança da Barragem-PSB, das Inspeções de Segurança Regular e Especial, da Revisão Periódica de Segurança de Barragem e do Plano de Ação de Emergência para Barragens de 
Mineração-PAEBM, conforme art. $8^{\circ}, 9^{\circ}, 10,11$ e 12 da Lei $n^{\circ} 12.334$ de 20 de setembro de 2010, que determina a Política Nacional de Segurança de Barragens - PNSB.

É de competência da ANM fiscalizar as atividades de pesquisa e lavra para o aproveitamento mineral e a segurança das barragens destinadas à disposição de rejeitos resultantes destas atividades, desenvolvidas com base em títulos outorgados pela própria autarquia e pelo Ministério de Minas e Energia - MME. A Lei no 12.334, de 20 de setembro de 2010, que estabeleceu a PNSB criou também o Sistema Nacional de Informações sobre Segurança de Barragens-SNISB. Considerando que o PSB é um instrumento da PNSB e que cabe ao empreendedor elaborá-lo e implementá-lo, incluindo, quando exigido pelo órgão fiscalizador, o PAEBM, nos termos dos arts. 8ㅇ, 11으 e 12 ㅇ da referida Lei.

A Lei no 12.334/2010, em seu parágrafo único do art. 1으, informa que será aplicada a barragens destinadas à acumulação de água para quaisquer usos, à disposição final ou temporária de rejeitos e à acumulação de resíduos industriais que apresentem pelo menos uma das seguintes características: I - altura do maciço, contada do ponto mais baixo da fundação à crista, maior ou igual a 15m (quinze metros); II capacidade total do reservatório maior ou igual a $3.000 .000 \mathrm{~m}^{3}$ (três milhões de metros cúbicos); III reservatório que contenha resíduos perigosos conforme normas técnicas aplicáveis; IV - categoria de dano potencial associado, médio ou alto. Estas informações estão contidas no quadro 1.

Quadro 1- Critérios das barragens de mineração que se enquadram na PNSB.

\begin{tabular}{|l|l|}
\hline CRITÉRIOS PARA SE ENQUADRAR NA PNSB & $>=15$ Metros \\
\hline Altura & $>=3$ milhões $\mathrm{m}^{3}$ \\
\hline Relume & $=$ Perigoso \\
\hline Dano Potencial Associado & $=$ Médio ou Alto \\
\hline
\end{tabular}

\section{Classificação das barragens de mineração}

Segundo o Art. 5ㅇ, Portaria № 70.389, de 17 de maio de 2017, as barragens de mineração serão classificadas pela ANM em consonância com o art. 70 da Lei no 12.334/2010 de acordo com o quadro de classificação quanto à Categoria de Risco- CRI e ao Dano Potencial Associado-DPA, nas classes A, B, C, D e E.

Quadro 2: Classe das barragens de mineração.

\begin{tabular}{|l|l|l|l|}
\hline \multirow{2}{*}{ Categoria de Risco } & \multicolumn{3}{|l|}{ Dano Potencial Associado } \\
\cline { 2 - 4 } & ALTO & MÉDIO & BAIXO \\
\hline ALTO & A & B & C \\
\hline MÉDIO & B & C & D \\
\hline BAIXO & B & C & E \\
\hline
\end{tabular}

A Matriz do quadro 2 relaciona a classificação quanto à CRI e ao DPA, com o objetivo de estabelecer a necessidade de elaboração do Plano de Ação de Emergência para Barragens de Mineração - PAEBM, a periodicidade das Inspeções de Segurança Regular- ISR, as situações em que deve ser realizada obrigatoriamente Inspeção de Segurança Especial - ISE, e a periodicidade da Revisão Periódica de Segurança de Barragem - RPSB.

DPA: refere-se ao dano que pode ocorrer devido ao rompimento ou mau funcionamento de uma barragem, independentemente da sua probabilidade de ocorrência, a ser graduado de acordo com as perdas 
de vidas humanas, saúde coletiva, impactos sociais, econômicos e ambientais. São consideradas Classe A barragens com Dano Potencial Associado Alto- DPAA e Categoria de Risco Alta- CRA. Na Classe E, estão as barragens com Dano Potencial Associado Baixo- DPAB e Categoria de Riso Baixo - CRB. Esta divisão segue o SNISB.

\section{Plano de Ação de Emergência para Barragens de Mineração- PAEBM}

O capítulo VI da portaria № 70.389, de 17 de maio de 2017, cuja versão atualizada constam as retificações de 05/06/2017, 10/11/2017 e resolução ANM no 13 de 08/08/2019, trata do PAEBM, onde informa que este deverá ser elaborado para todas as barragens abrangidas pela PNSB. O documento físico do PAEBM deverá ter capa vermelha e o nome da barragem em destaque, visando fácil localização no momento de sinistro e deverá estar em local de fácil acesso. Devem ser entregues cópias físicas do PAEBM para as prefeituras e aos órgãos de defesa civil.

Ainda segundo portaria supracitada, cabe ao empreendedor da barragem de mineração, dentre outras obrigações, providenciar a elaboração do PAEBM, disponibilizar as informações de ordem técnica à Defesa Civil, às prefeituras e demais instituições indicadas pelo governo municipal. Promover treinamentos internos, no máximo a cada seis meses, e manter os respectivos registros das atividades. Apoiar e participar de simulados de situações de emergência. Designar formalmente o coordenador do PAEBM e seu substituto. Possuir equipe de segurança da barragem capaz de detectar, avaliar e classificar as situações de emergência em potencial, de acordo com os níveis de emergência e declarar, quando for o caso, situaação de emergência para executar as ações descritas no PAEBM.

Desta forma, as empresas que operam barragens de mineração inseridas na PNSB possuem obrigatoriedade para entregar o PAEBM à ANM, com definições de ações imediatas a serem tomadas em caso de rompimento e com descrição das possíveis consequências ambientais, econômicas e de saúde coletiva. O PAEBM tem como objetivo mitigar os danos causados às comunidades, propriedades e ecossistemas atingidos em caso de rompimento de barragem. O não cumprimento das obrigações previstas sujeitará o infrator as penalidades estabelecidas no art.10 da Resolução ANM no 13/2019 que vê como medida preventiva a interdição da barragem.

\section{MATERIAIS E MÉTODOS}

\section{Área de estudo: a escolha do principal município minerador do Brasil}

A área de estudo da pesquisa focalizou o município de Parauapebas, visto que este está assentado na maior província mineral do planeta, a Serra dos Carajás (CORDANI et al., 2019). A Tabela 1 evidencia que Parauapebas desponta como principal município brasileiro de base mineral e, portanto, como importante protagonista no superávit na balança comercial do Brasil (PEREIRA et al., 2020). Parauapebas está localizado no estado do Pará, região Norte do país, na Amazônia Legal Brasileira. 
Tabela 1: Classificação dos municípios brasileiros quanto a produção mineral.

\begin{tabular}{|l|l|l|}
\hline Municípios do Brasil de maior produção mineral & Valor de operação em R\$ (2019) \\
\hline 1 & Parauapebas - PA & $32.451 .112 .676,36$ \\
\hline 2 & Canaã dos Carajás - PA & $20.533 .818 .313,53$ \\
\hline 3 & Congonhas - MG & $8.324 .531 .044,22$ \\
\hline 4 & Itabira - MG & $6.996 .612 .255,70$ \\
\hline 5 & Marabá - PA & $6.261 .539 .267,92$ \\
\hline 6 & Nova Lima - MG & $5.457 .631 .675,75$ \\
\hline 7 & Conceição do Mato Dentro - MG & $5.168 .630 .440,60$ \\
\hline 8 & São Gonçalo do Rio Abaixo - MG & $4.555 .012 .584,97$ \\
\hline 9 & Paracatu - MG & $3.467 .366 .908,52$ \\
\hline 10 & Itabirito - MG & $2.955 .236 .073,34$ \\
\hline 11 & Mariana - MG & $2.330 .255 .905,24$ \\
\hline 12 & Brumadinho - MG & $2.139 .980 .189,39$ \\
\hline
\end{tabular}

Fonte: ANM (2019).

Parauapebas possui a extensão de 6.886,208 km², com uma população estimada em 2019 de 208,273 habitantes e possui o 2으 maior PIB do estado do Pará. Sua principal atividade econômica é a mineração. Este município está a $719 \mathrm{~km}$ de distância da capital Belém. A figura 1 aponta as barragens de mineração localizadas em Parauapebas que estão classificadas pela ANM segundo o DPA à sua operação.

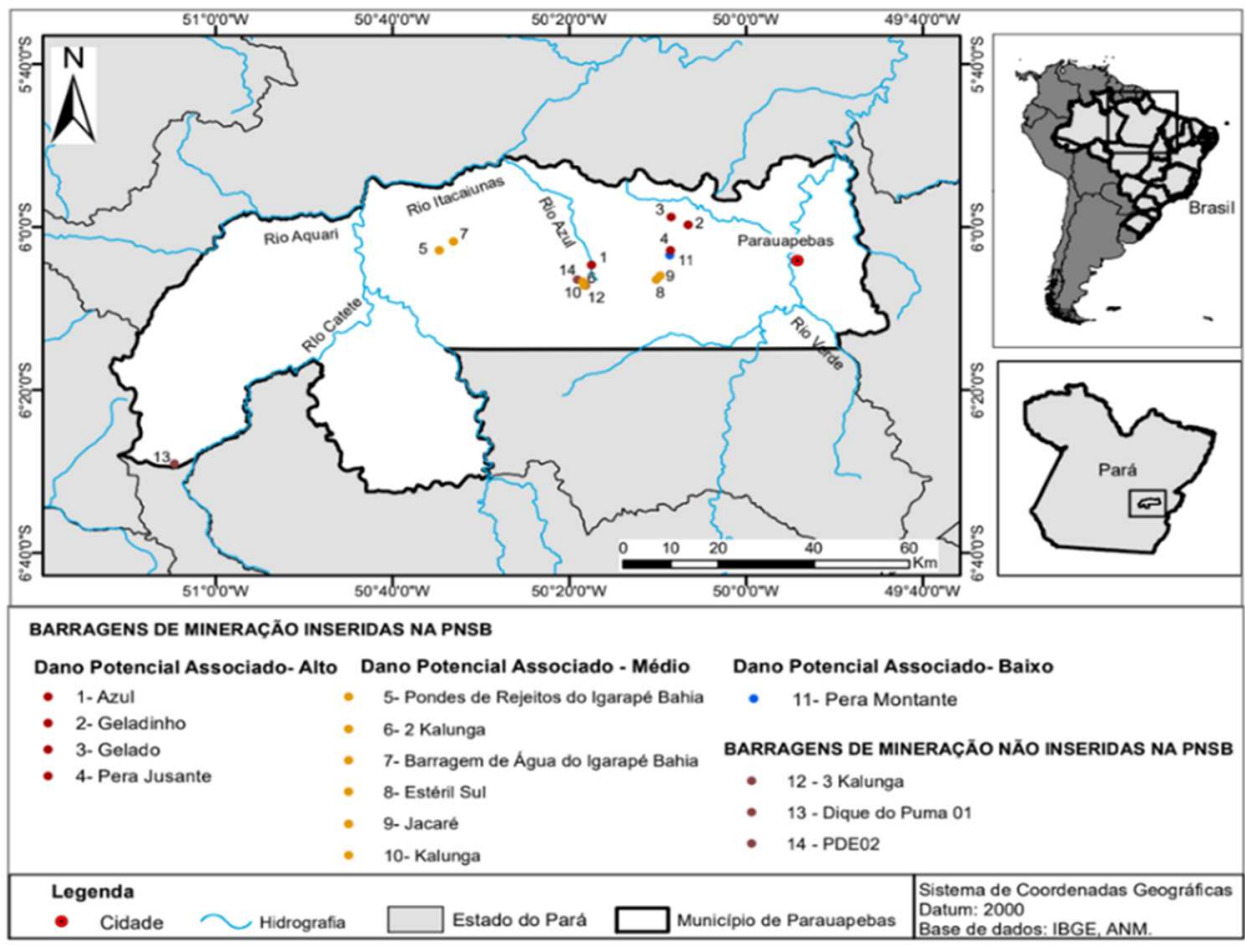

Figura 1: Mapa de localização das barragens no município de Parauapebas.

Para este estudo, as barragens de rejeitos escolhidas foram aquelas classificadas pela ANM com DPAA, denominadas como: Azul, Geladinho, Gelado e Pera Jusante, que estão inseridas na PNSB.

\section{Levantamento e análise de dados}

A pesquisa desenvolveu-se sob vasto arcabouço teórico de artigos científicos que versaram sobre o tema. Os dados secundários referentes às barragens de contenção de rejeitos da mineração foram coletados através de bancos de dados da ANM, do Sistema de Gestão de Segurança de Barragem de Mineração- SIGBM, onde estão disponibilizadas informações sobre a legislação vigente no Brasil, classificação dos municípios 
brasileiros quanto a produção de riqueza mineral, caracterização das barragens de rejeitos, dentre outras. As imagens de satélite das quatro barragens localizadas no município de Parauapebas foram adquiridas através do Google Maps. Os dados que tratam sobre os Danos Potenciais Associados aos riscos e impactos causados por rompimento de barragem de mineração foram adquiridos através do sítio eletrônico da Fundação Oswaldo Cruz - Fiocruz e foram considerados os danos, doenças e agravos decorrentes dos desastres ocorridos em Mariana e Brumadinho.

\section{RESULTADOS E DISCUSSÃO}

Com o intuito de facilitar a análise e discussão dos dados, a figura 2 apresenta a localização espacial das quatro barragens de rejeitos em operação, classificadas como DPAA.
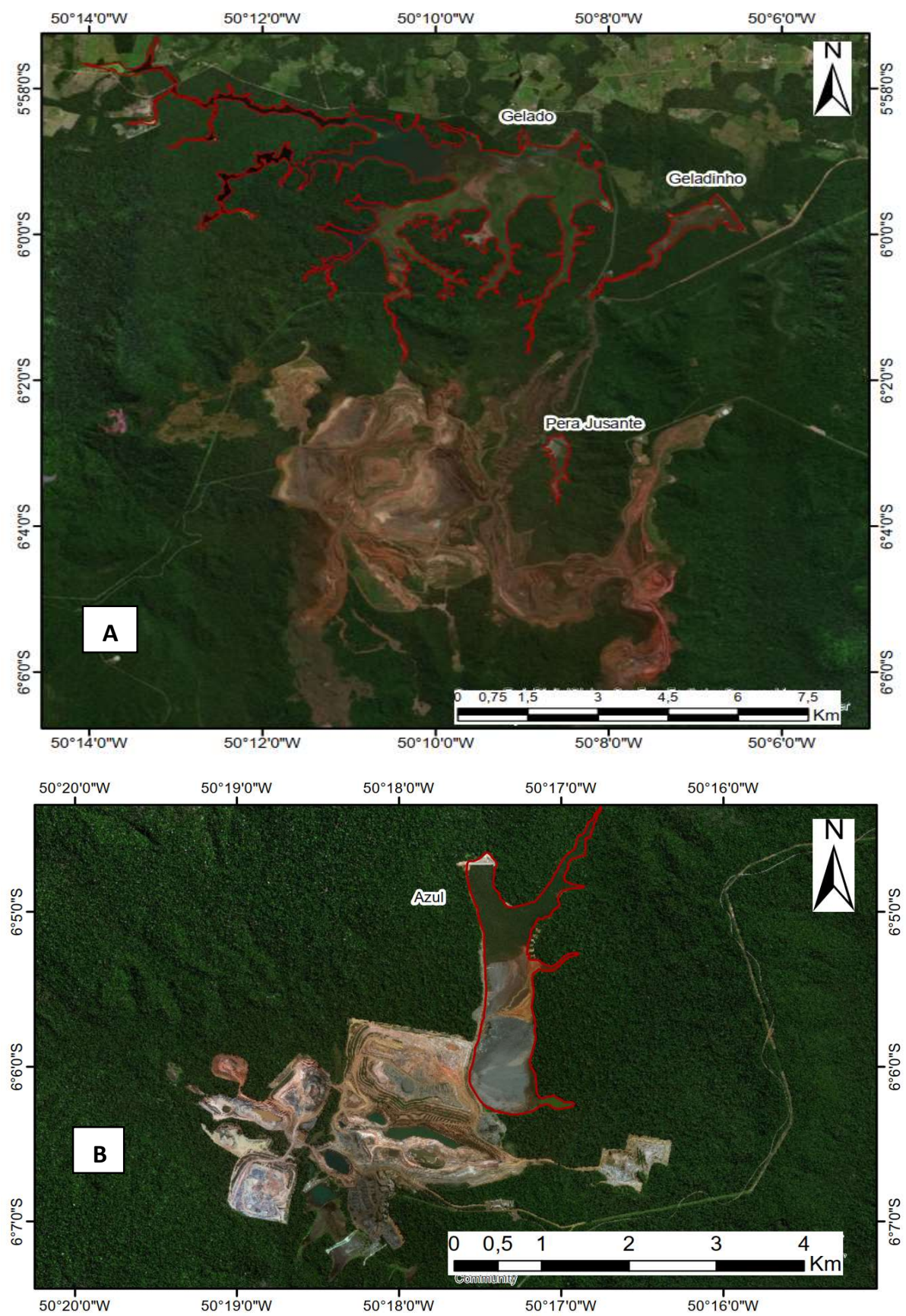

Figura 2: Localização espacial das Barragens classificadas como DPAA. Fonte: Google Maps (2020). A) Barragens Gelado, Geladinho e Pera Jusante. B) Barragem Azul. 
Segundo dados da ANM, nas barragens Azul, Geladinho e Pera Jusante foram declaradas cerca de 100 pessoas ocupando permanentemente a jusante. Na Barragem Gelado, o número de pessoas é maior, cerca de 1.000 habitantes próximos a jusante. Deste modo, vidas humanas poderão ser atingidas em caso de rompimento.

As quatro barragens em análise possuem impacto ambiental significativo, visto que a área próxima a jusante da barragem apresenta área de interesse ambiental relevante ou áreas protegidas em legislações específicas, excluídas as Áreas de Preservação Permanentes - APPs. As três barragens: Azul, Geladinho e Gelado possuem impactos socioeconômicos médios, pois existe moderada concentração de instalações residenciais, agrícolas ou de infraestrutura de relevância socioeconômico-cultural. A barragem Pera Jusante possui impacto socioeconômico baixo, pois existe pequena concentração de instalações residenciais e agrícolas. Todas as barragens em análise possuem o PAEBM, exceto a barragem Azul, cujo PAEBM ainda se encontra em fase de elaboração.

A tabela 2 apresenta a caracterização das quatro barragens de Parauapebas que apresentam DPAA, onde estão dispostas informações referentes à o principal minério extraído, método construtivo, altura, categoria de risco e classe da barragem.

Tabela 2: Caracterização das barragens de rejeitos de mineração de Dano Potencial Associado Alto - DPAA do município de Parauapebas.

\begin{tabular}{|l|l|l|l|l|l|l|}
\hline $\begin{array}{l}\text { Barragem } \\
\text { mineração }\end{array}$ & $\begin{array}{l}\text { Minério } \\
\text { principal }\end{array}$ & Método construtivo & $\begin{array}{l}\text { Altura } \\
(\mathrm{m})\end{array}$ & Volume $(\mathrm{m} 3)$ & $\begin{array}{l}\text { Categoria } \\
\text { risco }\end{array}$ & de \\
\hline Azul & Manganês & $\begin{array}{l}\text { Alteamento a } \\
\text { jusante }\end{array}$ & 32,0 & $12.995 .662,00$ & Baixa & B \\
\hline Geladinho & Ferro & Etapa Única & 24,0 & $6.544 .459,00$ & Baixa & B \\
\hline Gelado & Ferro & $\begin{array}{l}\text { Alteamento a } \\
\text { jusante }\end{array}$ & 34,0 & $141.187 .217,00$ & Baixa & B \\
\hline Pera Jusante & Ferro & Etapa Única & 51,0 & $4.402 .573,00$ & Baixa & B \\
\hline
\end{tabular}

É válido mencionar que, devido à intensificação da atividade minerária no município de Parauapebas, está ocorrendo gradativamente nas quatro barragens em operação o aumento do volume dos rejeitos, o que eleva a necessidade de monitoramento e fiscalização nesses empreendimentos para evitar rompimentos.

Tendo como referência os desastres recentes ocorridos nos municípios de Mariana e Brumadinho, em Minas Gerais, o quadro 3 apresenta através de dados da Fiocruz, os Danos Potenciais Associados aos riscos e impactos causados por rompimento de barragem de mineração.

Foi sistematizado o conjunto de riscos e impactos, bem como efeitos sobre a saúde que se sobrepõem. Os dados revelaram uma multiplicidade de efeitos sobre a saúde humana, combinando o agravamento e ampliação de doenças preexistentes com o surgimento de novas, em um cenário de sobreposição de riscos, doenças e danos (NISHIJIMA, 2020). A complexidade desses tipos de eventos requer prévia delineação governamental, visto que estes desastres resultam em interrupção abrupta e grave no cotidiano das comunidades que vivem no entorno dos empreendimentos minerários (ZORZAL et al., 2019).

Nessas circunstancias, o bem-estar social é afetado, podendo envolver perdas de vidas, perdas econômicas, danos ambientais e à saúde coletiva, através de agravos e doenças que podem resultar em óbitos imediatos, assim como também em doenças crônicas e de desdobramentos de longo prazo, de modo 
a sobrecarregar a estrutura de saúde pública do município afetado (FREITAS et al., 2019). Além disso, essas tragédias ambientais, por envolverem contaminação de rios, florestas, animais e seres humanos exigem decisões planejadas para serem executadas em condições de urgência, como um plano de contingenciamento efetivo, para administrar as incertezas, cessar ou diminuir as exposições e riscos, bem como mitigar os danos ocorridos (DIAS et al., 2018; ROMÃO et al., 2019).

Quadro 3: Danos Potenciais Associados aos riscos e impactos causados pelo desastre.

\begin{tabular}{|c|c|c|c|c|c|c|c|c|c|c|}
\hline \multirow[b]{2}{*}{ RISCOS E IMPACTOS } & \multicolumn{10}{|c|}{ POTENCIAIS DANOS À SAÚDE COLETIVA } \\
\hline & 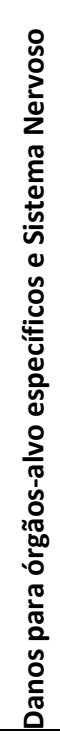 & 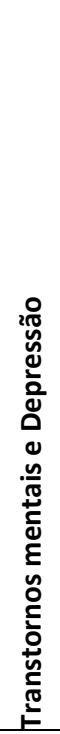 & 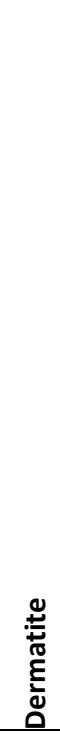 & 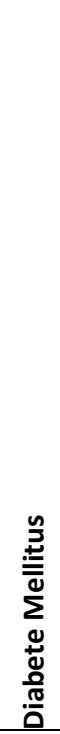 & 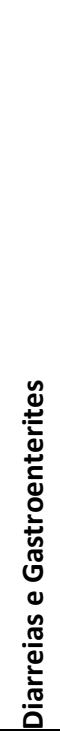 & 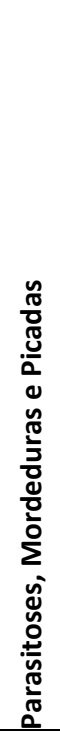 & 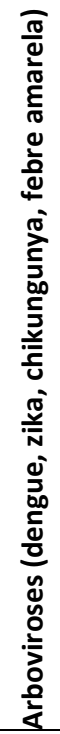 & 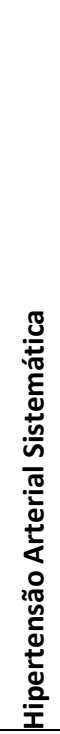 & 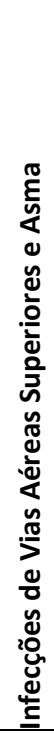 & $\begin{array}{l}\frac{0}{0} \\
\frac{\pi}{0} \\
\frac{d}{v} \\
\frac{1}{4}\end{array}$ \\
\hline Contaminação de rios e sedimentos & $\mathbf{X}$ & $\mathbf{x}$ & $\mathbf{X}$ & $\mathbf{X}$ & $\mathbf{x}$ & & & $\mathbf{X}$ & & \\
\hline Contaminação do solo e atmosférica & $\mathbf{x}$ & $\mathbf{x}$ & $\mathbf{X}$ & $\mathbf{x}$ & & & & $\mathbf{x}$ & $\mathbf{X}$ & \\
\hline Alteração dos ciclos de vetores, hospedeiros e reservatórios & & & & & $\mathbf{x}$ & $\mathbf{x}$ & $\mathbf{x}$ & & & \\
\hline Degradação dos aspectos sanitários e da qualidade de vida & & $\mathbf{x}$ & & & $\mathbf{x}$ & $\mathbf{x}$ & & $\mathbf{x}$ & $\mathbf{x}$ & $\mathbf{x}$ \\
\hline Impactos Socioeconômicos & & $\mathbf{X}$ & & $\mathbf{X}$ & & & & $\mathbf{x}$ & & $\mathbf{x}$ \\
\hline
\end{tabular}

Para além da experiência dos municípios afetados nas suas ações pós-desastre, bem como dos danos ao meio ambiente e saúde física e mental dos atingidos a médio e longo prazos, desastres como esses lançam uma série de desafios para a indústria minerária e para a gestão pública do Brasil para que não ocorram outras tragédias semelhantes.

Entretanto, os grandes empreendimentos mineradores do país e do estado do Pará estão em setores econômicos intensivos em recursos naturais e primários da cadeia produtiva (RIBEIRO et al., 2018). O crescimento destas empresas tem como principal contraponto a intensificação e ampliação dos impactos relacionados às atividades extrativas minerárias (CASTRO, 2019). Tais empresas expandem sua produção e exportação de commodities até que as minas sejam exauridas, enquanto que a degradação ambiental e danos à saúde coletiva incidem localmente (CORRÊA, 2018).

Isto posto, pode-se afirmar que o Município de Parauapebas, por ter sua economia atrelada e dependente da atividade minerária, tende a ficar mais exposto às externalidades causadas pela atividade da mineração. As quatro barragens de rejeitos consideradas com DPAA em análise, são de fato potenciais degradadoras ambientais e ameaçam o bem-estar e a saúde das comunidades locais.

Nessa perspectiva, cabe afirmar que a intensiva exploração da riqueza mineral tem sido 
responsabilizada por graves problemas ambientais e sociais que foram historicamente impostos à região Amazônica (PALHETA et al., 2018). O Pará é o maior produtor mineral do País, com jazidas de minérios como ferro, cobre, níquel, bauxita, caulim, manganês, silício e ouro. A mineração industrial está presente em 61 municípios paraenses, sendo uma atividade produtiva consolidada nesta região, cujos resultados da atuação das indústrias mineradoras possuem capacidade de gerar reflexos socioeconômicos positivos localmente (LOBATO, 2019). Entretanto, na prática, a produção de riqueza proveniente da indústria minerária na Amazônia não possui relação direta com o desenvolvimento socioeconômico, ambiental e de saúde pública na região (ROCHA et al., 2019).

As empresas mineradoras devem buscar novos métodos e práticas na mineração que conciliem a eficiência econômica com a eficiência ambiental (LIU et al., 2019). Uma excelente alternativa para a resolução dessa problemática é o beneficiamento do minério de ferro à seco, que não gera rejeitos e que, portanto, não utiliza barragens de contenção. Em Canaã dos Carajás, no sudeste do Pará, há o Complexo S11D Eliezer Batista, inaugurado pela da Vale em 2016 que utiliza esse método. Entretanto, por ser ainda uma solução inovadora, apresenta elevados custos de produção que comprometem substancialmente as margens de lucro das empresas mineradoras, inibindo sua difusão para todo o setor (SUN et al., 2019).

Atualmente, as companhias de mineração no mundo promovem estudos de práticas que visam equilibrar a exploração mineral e o meio ambiente (RIBEIRO e tal., 2018). Estratégias mais inteligentes e compensadoras são aquelas que aproveitam os rejeitos, valorizam os subprodutos e neutralizam as poluições com os resíduos da própria mineração (TAYEBI-KHORAMI et al., 2019).

\section{CONCLUSÕES}

Diante do cenário de crimes ambientais deflagrados por práticas danosas da indústria mineral, o Brasil tornou-se um exemplo de falha de infraestrutura com consequências catastróficas. $\mathrm{O}$ impacto relativo à poluição, degradação ambiental e danos à saúde coletiva é denso, de longo prazo e difícil de quantificar.

Para a melhoria no gerenciamento dessas atividades, mitigação de colapsos de barragens e para evitar acidentes semelhantes torna-se imperativo maior rigor da gestão pública, bem como suscitar questionamentos científicos sobre o processo de exploração dos recursos minerais ou as práticas socioespaciais desenvolvidas pelas mineradoras. No entanto, diante do contexto apresentado, há o predomínio, com anuência do Estado, das práticas danosas da mineração para com o meio ambiente. Há também o déficit de pesquisas na Amazônia e no país que questionem e que busquem compreender os problemas socioambientais provocados pelo processo produtivo das Indústrias minerárias locais.

Assim sendo, apesar dos desastres recentes ocorridos no país, a fiscalização para os cumprimentos legais e atendimento às normas estabelecidas ainda é limitada e muito dependente do monitoramento das próprias mineradoras, o que aumenta ainda mais o potencial de degradação ao ambiente e a saúde coletiva face à exploração de minério. Mediante análise da legislação brasileira sobre barragens de rejeitos da mineração foi percebido que está possui lacunas para o consentimento de licenciamentos frágeis e com precário poder fiscalizatório do Estado. 
Há ainda a existência tacanha e ineficaz do PAEBM, cuja obrigatoriedade por lei é recente e que possui dificuldades para ser executado com eficiência, haja vista que atualmente, no estado do Pará, inexistem condições objetivas e eficazes de garantir a segurança destas barragens, pois há um intrincado cenário político e econômico desfavorável ao cumprimento das normas, aliado ao exíguo número de fiscais capacitados para atuar no controle e fiscalização de forma preventiva sobre condições de riscos ou falhas nessas estruturas.

Faz-se necessário que o Estado brasileiro - em consonância com a preocupação da comunidade científica e de Governos internacionais respeitosos - esteja comprometido com a imperiosa necessidade de proteção ao meio ambiente e à vida humana. Que as governanças das esferas federais, estaduais e municipais estejam abertas às novas possibilidades que busquem soluções mais sustentáveis para que a atividade minerária, que constitui em importante geradora de divisas para o estado do Pará, possa também ser um exemplo de processo produtivo onde os recursos de produção sejam otimizados com respeito ao meio ambiente e à população que reside e trabalha no entorno desses empreendimentos.

\section{REFERÊNCIAS}

AIRES, U. R. V.; SANTOS, B. S. M.; COELHO, C. D.; SILVA, D. D.; CALIJURI, M. L.. Changes in land use and land cover as a result of the failure of a mining tailings dam in Mariana, MG, Brazil. Land Use Policy, v.70, p.63-70, 2018. DOI: https://doi.org/10.1016/j.landusepol.2017.10.026

ANM. Agência Nacional de Mineração. Anuário Mineral Brasileiro. Maiores Arrecadadores-operação. Brasília: ANM, 2019.

ANM. Agência Nacional de Mineração. Sistema de Gestão de Segurança de Barragem de Mineração. Brasília: ANM, 2019.

BURRITT, R. L.; CHRIST, K. L.. Water risk in mining: Analysis of the Samarco dam failure. Journal of Cleaner Production, v.178, p.196-205, 2018. DOI:

https://doi.org/10.1016/j.jclepro.2018.01.042

CASTRO, R. A.. Os incentivos do Programa Grande Carajás às grandes empresas e as repercussões em comunidades rurais. HOLOS, v.6, 2019. DOI:

https://doi.org/10.15628/holos.2019.5573

CORDANI, U. G.; JULIANI, C.. Potencial mineral da Amazônia. Revista de Estudios Brasileños, v.6, n.11, p.91108, 2019. DOI: https://doi.org/10.14201/reb201961191108

CORRÊA, A. E. C.. Global players, mineração e crise: o crime da produção de valor. Lutas Sociais, v.22, n.41, 268-279, 2018.

ALMEIDA, L. G.; CORTELETTI, R. C.; DUTRA, L. F.. Metodologia para classificação do Índice de Perigo (IP) em Barragens de rejeitos da Mineração. Geociências, v.38, n.3, p.765-780, 2020. DOI: https://doi.org/10.5016/geociencias.v38i3

MORAES, S. L.; RIBEIRO, T. R.. Brazilian iron ore and production of pellets. Mineral Processing and Extractive Metallurgy Review, v.40, n.1, p.16-23, 2019. DOI: https://doi.org/10.1080/08827508.2018.1481056

SOUZA, P. A.. Open data could have helped us learn from another mining dam disaster. Scientific Data, v.6, n.1, p.54, 2019. DOI: https://doi.org/10.1038/s41597-019-0063-0

DIAS, C. A.; COSTA, A. S. V.; GUEDES, G. R.; MATOS UMBELINO, G. J.; SOUSA, L. G.; ALVES, J. H.; SILVA, T. G. M.. Impactos do rompimento da barragem de Mariana na qualidade da água do rio Doce. Revista Espinhaço, p.21-35, 2018.

DIOS, S. A.; COSENZA, J. P.. Corporate social responsibility, multinational companies and economic globalization. Dimensión Empresarial, v.17, n.4, 2019. DOI: https://doi.org/10.15665/dem.v17i4.1964

CARMO, F. F.; KAMINO, L. H. Y.; JUNIOR, R. T.; CAMPOS, I. C.; CARMO, F. F.; SILVINO, G.; CASTRO, K. J. S. X.; MAURO, M. L.; RODRIGUES, N. U. A.; MIRANDA, M. P. S.; PINTO, C. E. F.. Fundão tailings dam failures: the environment tragedy of the largest technological disaster of Brazilian mining in global context. Perspectives in Ecology and Conservation, v.15, n.3, p.145-151, 2017. DOI: https://doi.org/10.1016/j.pecon.2017.06.002

SANT'ANA FILHO, J. N. S. A.; SILVA, S. N.; SILVA, G. C.; MENDES, J. C.; PEIXOTO, R. A. F.. Technical and environmental feasibility of interlocking concrete pavers with iron ore tailings from tailings dams. Journal of Materials in Civil Engineering, v.29, n.9, p.04017104, 2017. DOI: https://doi.org/10.1061/(ASCE)MT.1943-5533.0001937

FREITAS, C. M. D.; BARCELLOS, C.; ASMUS, C. I. R. F.; SILVA, M. A. D.; XAVIER, D. R.. Samarco em Mariana à Vale em Brumadinho: desastres em barragens de mineração e Saúde Coletiva. Cadernos de Saúde Pública, v.35, p.e00052519, 2019. DOI: https://doi.org/10.1590/0102-311X00052519 FREITAS, C. M. D.; BARCELLOS, C.; HELLER, L.; LUZ, Z. M. P 
D.. Mining dam disasters: lessons from the past for reducing current and future risks. Epidemiologia e Serviços de Saúde, v.28, n.1, 2019. DOI: http://dx.doi.org/10.5123/s1679$\underline{49742019000100020}$

FREITAS, C. M. D.; SILVA, M. A. D.; MENEZES, F. C. D.. O desastre na barragem de mineração da Samarco: fratura exposta dos limites do Brasil na redução de risco de desastres. Ciência e Cultura, v.68, n.3, p.25-30,2016. DOI: http://dx.doi.org/10.21800/2317-66602016000300010

GARCIA, L. C.; RIBEIRO, D. B.; OLIVEIRA, F. R.; OCHOAQUINTERO, J. M.; LAURANCE, W. F.. Brazil's worst mining disaster: corporations must be compelled to pay the actual environmental costs. Ecological Applications, v.27, n.1, p.59, 2017. DOI: https://doi.org/10.1002/eap.1461

GEROTTO, G.; PISANO, V.; DEMAJOROVIC, J.; ALEDO, A.; SANTIAGO, A. L.. Impacto social da mineração: uma comparação entre a percepção da empresa e a da comunidade. Contextus: Revista Contemporânea de Economia e Gestão, v.17, n.3, 2019. DOI: https://doi.org/10.19094/contextus.v17i3.42618

GEVORKYAN, A. V.. Exchange market pressure and primary commodity-exporting emerging markets. Applied Economics, v.51, n.22, p.2390-2412, 2019. DOI: https://doi.org/10.1080/00036846.2018.1545077

HAURADOU, G. R.; AMARAL, M. V. B.. Mining in Brazilian Amazon: Aspects of Capital Presence and Advancement in the Region. Revista de Políticas Públicas, v.23, n.1, p.402420, 2019. DOI: https://dx.doi.org/10.18764/217865

HINMAN, P.. Brazil: New vale mine disaster is one more corporate failure. Green Left Weekly, n.1207, p.15, 2019.

LIU, X.; GUO, P.; GUO, S.. Assessing the eco-efficiency of a circular economy system in China's coal mining areas: Emergy and data envelopment analysis. Journal of Cleaner Production, v.206, p.1101-1109, 2019. DOI: https://doi.org/10.1016/i.jclepro.2018.09.218

LOBATO, M. M.. A Dinâmica na Fronteira Paraense e os Territórios do Capital: Pecuária, extração madeireira e mineração. Formação, v.26, n.47, 2019. DOI: https://doi.org/10.33081/formacao.v26i47.5600

MEYER, V.; MAMÉDIO, D. F.; ADRIÃO, M.. The Fundão Tailings Dam Catastrophe: How Unsustainable Practices and Sloppy Management Led to the Disaster. In: UNIVERSITIES AND SUSTAINABLE COMMUNITIES: MEETING THE GOALS OF THE AGENDA 2030. Anais. 2020. p.385-399. Cham: Springer, DOI: https://doi.org/10.1007/978-3-030-30306-8 23

\section{NEGRÃO, M. P.. Renda e atividades econômicas na} Amazônia. 2019.

NISHIJIMA, M.; ROCHA, F. F.. An economic investigation of the dengue incidence as a result of a tailings dam accident in Brazil. Journal of Environmental Management, v.253, p.109748, 2020. DOI: https://doi.org/10.1016/j.jenvman.2019.109748

PALHETA, J. M.; NETO, A. C. O.. Por uma Territorialização na Amazônia Paraense. Revista NERA, v.42, p.354-372, 2018.
PELICICE, F. M.. Weak Democracies, Failed Policies, and the Demise of Ecosystems in Poor and Developing Nations. Tropical Conservation Science, v.12, 2019. DOI: https://doi.org/10.1177\%2F1940082919839902

PEREIRA, M. M.; AMORIM, G. V. B. L.; CRISPIM, D. L.; FERNANDES, L. L.. Sustainability analysis in the city of Parauapebas-Amazon, Pará, Brazil. Research, Society and Development, v.9, n.3, 2020.

DOI: https://doi.org/10.33448/rsd-v9i3.2343

RIBEIRO, M. L.; COSTA, L. D. S.. O Processo de Industrialização e as políticas Industriais no Brasil. Orbis Latina, v.8, n.2, p.216-229, 2018.

RIBEIRO, J. C. J.; NASCIMENTO, L. C.. A Mineração no Estado do Pará e as Barragens de Rejeito: o paradigma entre a exploração e os impactos negativos decorrentes. In: CONGRESSO INTERNACIONAL DE DIREITO AMBIENTAL, 5. Anais. 2018.

ROCHA, N., TRINDADE, J. R. B.. O comércio exterior brasileiro e a questão regional: elementos da dinâmica econômica do estado do Pará nas duas últimas décadas. Cadernos CEPEC, v.2, p.1-6, 2019.

ROMÃO, A.; BARCELLOS, C.; XAVIER, D. R.; SALDANHA, R.; GRACIE, R.; PASCOAL, V.. Nota técnica: avaliação dos impactos do desastre de Brumadinho sobre a saúde. Rio de Janeiro: Observatório de Clima e Saúde, 2019.

SEVERO, E. M. F.; SOUSA, H. J. C.. Safety in Rejects Dams: a major challenge. In World Conference on Qualitative Research, v.1, p.340-343, 2019.

SCHORN, Y. A. G.; FENGLER, R. Z.. Barragens de Rejeitos de Minérios e as Condicionantes Geotécnicas Envolvidas. Revista GEDECON-Gestão e Desenvolvimento em Contexto, v.7, p.34-40, 2019.

DOI: http://dx.doi.org/10.33053/gedecon.v7i2.9427

SOMMER, J. M.; SHANDRA, J. M.; COBURN, C.. Mining exports flows, repression, and forest loss: a cross-national test of ecologically unequal exchange. Ecologically Unequal Exchange, Cham, p.167-193, 2019. DOI: https://doi.org/10.1007/978-3-319-89740-0 7

SOUZA, T. P.; SOUZA, R.; ALMEIDA, C. P. B.; ROSSETTO, M. Trabalhador de mineração: revisão dos aspectos relativos à silicose, doenças associadas e legislação vigente. Enfermería Comunitaria, 2019.

SUN, S.; ANWAR, S.. R\&D activities and FDI in China's iron ore mining industry. Economic Analysis and Policy, v.62, p.47-56, 2019. DOI: https://doi.org/10.1016/j.eap.2019.01.003

TAYEBI-KHORAMI, M.; EDRAKI, M.; CORDER, G.; GOLEV, A.. Re-thinking mining waste through an integrative approach led by circular economy aspirations. Minerals, v.9, n.5, p.286, 2019. DOI: https://doi.org/10.3390/min9050286

THOMÉ, R.; LAGO, T. M. O.. Ambiental, U. E. Barragens de rejeitos da mineração: o princípio da prevenção e a implementação de novas alternativas. Revista de Direito Ambiental, v.85, p.17-39, 2017. 
ZORZAL, M.; CAYRES, D. C.; SOUZA, L. A. M.. Desastre socioambiental e Termo de Transação e Ajustamento de Conduta (TTAC) como instrumento de política pública: o caso da barragem de Fundão, MG. Civitas-Revista de

Ciências Sociais, v.19, p.464-488, 2019.

DOI: https://doi.org/10.15448/1984-7289.2019.2.30227

A CBPC - Companhia Brasileira de Produção Científica (CNPJ: 11.221.422/0001-03) detém os direitos materiais desta publicação. Os direitos referem-se à publicação do trabalho em qualquer parte do mundo, incluindo os direitos às renovações, expansões e disseminações da contribuição, bem como outros direitos subsidiários. Todos os trabalhos publicados eletronicamente poderão posteriormente ser publicados em coletâneas impressas sob coordenação da Sustenere Publishing, da Companhia Brasileira de Produção Científica e seus parceiros autorizados. Os (as) autores (as) preservam os direitos autorais, mas não têm permissão para a publicação da contribuição em outro meio, impresso ou digital, em português ou em tradução. 